\title{
Friction and Wear Characteristics of 30CrMnSi-LD10-CS Under Constant Pressure
}

\author{
Jian Wu \\ Department of Mechanical \\ Engineering \\ Changshu Institute of Technology \\ Changshu, China \\ wujian123abc@163.com
}

\author{
$\mathrm{Xu} \mathrm{Li}$ \\ Educational Technology Center \\ Cheng Xian College \\ Southeast University \\ Nanjing, China
}

\author{
Te Li, Deli Liu, Lanzhong Guo \\ Department of Mechanical \\ Engineering \\ Changshu Institute of Technology \\ Changshu, China
}

\begin{abstract}
In order to investigate friction and wear characteristics of $30 \mathrm{CrMnSi}-\mathrm{LD10}-\mathrm{CS}$ friction pair, the friction and wear test rig was built to measure the friction torque and wear coefficient under low speed, and mathematical calculation was used to figure out the relationship between friction torque and wear coefficient. The results have shown that friction coefficient measured by the amount of wear increases with time, friction coefficient measured by torque decreases with time. The ratio of friction coefficient and wear coefficient of the designed friction pair has a certain logarithmic relationship with time.
\end{abstract}

Keywords-30CrMnSi-LD10-CS; Friction coefficient; Wear coefficient

\section{INTRODUCTION}

Sliding friction pair is a common form of movement pairs [1], which is usually used in piston cylinder with high-speed reciprocating motion, machine tool with medium speed and the elevator door lock with low speed. High speed friction pair is generally required for surface treatment, the main method is chrome plating, tin plating. The medium speed friction pair is usually needed to be strengthened treatment, the main method is annealing and quenching. The low speed moving pair has low load / speed ratio, and the anti-rust processing does not change the mechanical properties, so the wear of the low speed moving pair directly reflects the mechanical properties of the material itself [2-6].

The friction coefficient reflects the properties of the friction pair, which is only related to the material itself. The wear coefficient reflects the mechanical properties of the contact surface, and it has a great relationship with the performance of the contact surface. When two nominally mating surfaces are brought together, they touch at the tips of the higher asperities and the total area of intimate contact is determined by the deformation of the material in these regions under the applied load. In the early 1950s, Bowden and Tabor discovered the character and magnitude of the transfer of material between rubbing surfaces and their wear rate may show a similar dependence [7]. Based on these results, Archard worked out the theory of adhesive wear, which revealed the relationship between the wear with contact area, hardness, friction coefficient and applied load [8-10]. In 2009-2013, Cozza set a ball-cratering test and studied on friction and wear coefficient of coated systems with micro-scale refer to that theory [11-13].

This paper focuses on the friction performance of the aviation door locking device materials 30CrMnSi-LD10-CS, the build of friction wear experimental test platform was used to measure friction torque and the amount of wear, so that the relationship between friction coefficient and wear coefficient was discussed under the condition of low speed.

\section{THEORY}

In 1953, J F Archard [8] put forward a model later called Archard wear theorem, which described the relationship between wear volume with sliding distance, hardness and applied load.

$$
\frac{W}{S}=K \frac{F_{n}}{H}
$$

Where, $W$ is the wear volume, $S$ is the sliding distance; $H$ is the Brinell hardness of the soft material; $K$ is the friction coefficient caused by the wear. Considering the wear is a dynamic process, the wear volume and sliding distance is increasing with time, Equation (1) can be changed to:

$$
w_{v}(t)=\frac{K \cdot F_{n} \cdot S(t)}{H}
$$

Where $W_{v}(\mathrm{t})$ is the wear volume for the corresponding time, $S(\mathrm{t})$ is the sliding distance for the corresponding time.

For a round friction, the wear volume $W_{v}(\mathrm{t})$ is written as:

$$
w_{v}(t)=\pi r^{2} \Delta h(t)
$$

Where $r$ is the diameter of the round friction surface, $\Delta h(\mathrm{t})$ is the wear depth.

Take equation (3) into equation (2), wear increment can be calculated as: 


$$
\Delta h(t)=\frac{K \cdot F_{n} \cdot S(t)}{\pi r^{2} \cdot H}
$$

Therefore, based on the linear assumption, equation (4) is the equation of the wear loss under constant amplitude loading.

The friction coefficient $K$ is written as:

$$
K=\frac{\pi r^{2} \Delta h(t) \cdot H}{F_{n} \cdot S(t)}
$$

For the friction pair of pin disk structure, $S(\mathrm{t})=n 2 \pi R$, where $n$ is number of revolutions, $R$ is distance of the contact surface from the center of rotation. Positive load $F_{\mathrm{n}}=f / \mu$, where $f$ is friction force, $\mu$ is friction coefficient, so equation (5) can be written as:

$$
\frac{K}{\mu}=\frac{r^{2} \Delta h(t) \cdot H}{2 n T}
$$

Equation (6) shows the relationship between wear coefficient and friction coefficient, for a fixed friction pair, $R, h$ are constant. The relationship between wear coefficient and friction coefficient can be drawn with the measurement of $\Delta h$ (t), $N$, and torque $T$.

\section{EXPERIMENT}

Based on the theory mentioned above, the experiment aims to measure the friction torque and wear volume of aviation door locking device materials 30CrMnSi-LD10-CS, and the friction coefficient and wear coefficient of friction pairs under constant load were calculated. Material properties of $30 \mathrm{CrMnSi}-\mathrm{LD} 10-\mathrm{CS}$ is given in Table I. The applied load and the friction pair size is also given. The load $F_{\mathrm{n}}$ is applied on contacting surface, which contain the acting load $P$ and the weight of upper specimen $G$.

TABLE I. THE LOAD AND THE MECHANICAL PROPERTIES OF THE TEST MATERIAL

\begin{tabular}{c|c|c|c|c|c}
\hline \multirow{2}{*}{$\begin{array}{c}\text { Test } \\
\text { material }\end{array}$} & \multirow{2}{*}{$\begin{array}{c}\boldsymbol{F}_{\mathbf{n}} \\
/ \mathbf{K N}\end{array}$} & $\begin{array}{c}\text { Yield } \\
\text { strength } \\
/ \mathbf{M P a}\end{array}$ & $\begin{array}{c}\text { Strength } \\
\text { limit /MPa }\end{array}$ & $\begin{array}{c}\text { Brinell } \\
\text { Hardness } \\
\mathbf{/ N} / \mathbf{m m}^{2}\end{array}$ & $\begin{array}{c}\text { diagram/ } \\
\mathbf{~} \mathbf{m}\end{array}$ \\
\cline { 1 - 4 } LD10-CS & \multirow{2}{*}{10} & 75 & 490 & 1100 & 30 \\
\cline { 1 - 3 } & & 800 & 1200 & 3650 & 200 \\
\hline
\end{tabular}

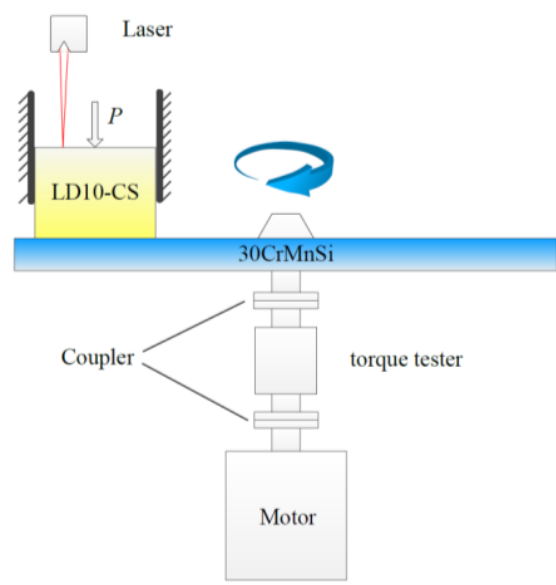

Figure 1. The principle of tester
In order to verify the above theoretical results, this paper mainly adopts the way of experimental study. The friction and wear test rig has been built and the structural schematic diagram is shown in figure 1 , which composition with laser measurement, frictional pair of $30 \mathrm{CrMnSi}-\mathrm{LD} 10-\mathrm{CS}$, torque meter and motor.

Test principle of testing rig is as follows: The motor is connected with the lower specimen $(30 \mathrm{CrMnSi})$ through a torque sensor. The upper specimen (LD10-CS) is fixed in the installing hole, which is ensure the position of the friction pair is constant during running, and a certain load $P$ is applied on the surface of the upper specimen. The laser measurement is placed directly over the upper specimen, which measured the top surface distance of the upper specimen (LD10-CS) in vertical direction.

During the running of the friction and wear testing rig, the motor drives the lower specimen $(30 \mathrm{CrMnSi})$ to rotate, the torque sensor measures the torque variation caused by the friction force. The laser measurement is used to measure the drop distance of the upper specimen (LD10-CS) caused by wear. The torque and friction torque information can be read and record by computer.

In order to obtain a larger amount of wear, the running time is set to $50 \mathrm{~h}$; the rotational speed is set to $7 \mathrm{rpm}$, the distance between the specimen and the center of rotation is $0.05 \mathrm{~m}$, the velocity of contacting friction pair is $0.037 \mathrm{~m} / \mathrm{s}$ by calculating. The torque and the laser measurement ranging are recorded every hour. The friction coefficient $\mu$ and wear coefficient $K$ can be obtained by friction force calculation equation and equation (5).

\section{RESULTS AND DISCUSSIONS}

\section{A. Torque measurement}

Torque is obtained by torque sensor, and the results are shown in Figure 2. The numbers of point in figure are the different between loads with unload torque. Owing to the recorded curve from $8 \mathrm{~h}$ to $50 \mathrm{~h}$, the fitting curve of friction torque tends is developed, which facilitates the numerical analysis.

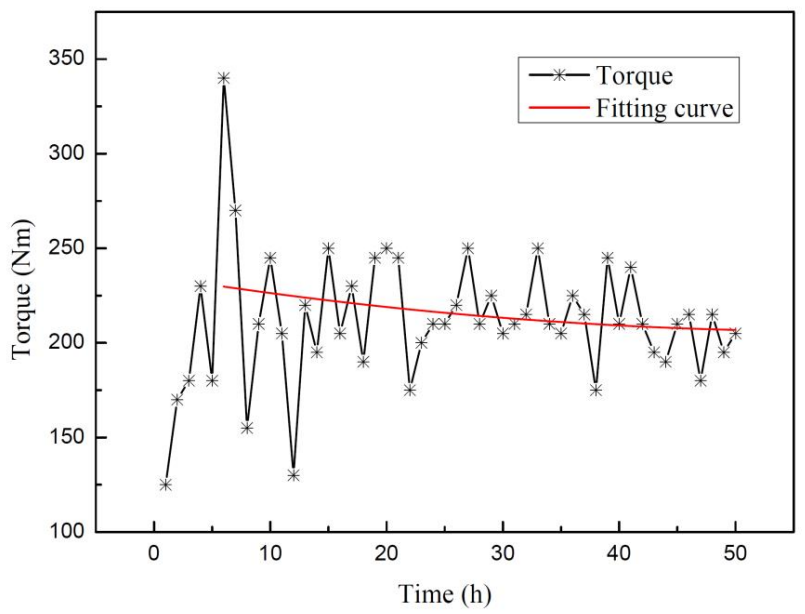

Figure 2. Results of torque measurement 
As it can be seen from upper figure that the value of torque is considerably fluctuant in the whole test phase, which is more obvious in the beginning and then gradually reduced, all of torque values are between $125 \mathrm{Nm}$ and $300 \mathrm{Nm}$. In the initial stage, the value of torque increases and is up to a maximum value, $342 \mathrm{Nm}$. When the experiment is conducted for 8 hours, the friction torque began to decline, and tends to be stable, large amount of data in between $200 \mathrm{Nm}$ and 250 $\mathrm{Nm}$. The followed data shows decreasing trend, the fitting curve in the figure indicates the variation trends, it is $213 \mathrm{Nm}$ when the wear test running to $50 \mathrm{~h}$.

The results show that friction value of metal materials friction pair (30CrMnSi-LD10-CS) and not constant. At the startup of the test rig, the obvious running-in occurs in the friction pair, the torque value keeps increasing. When the torque reached the maximum value, the friction came into the normal wear stage, and the torque tends to be stable. All of this process conforms to the general wear regularity. In this test, the torque gradually decreased because that the wear debris has played a part of lubrication, which is reduced the friction between the contact pair.

\section{B. Wear measurement}

The thickness of wear measured by laser range finder is shown in Figure 3. The distance of the upper specimen top surface is set to $0 \mu \mathrm{m}$ before running the motor. The numbers of point in figure are the measurement values with laser measurement. Owing to the recorded values, the fitting curve of wear distance tends is developed, which fitting formula using polynomial.

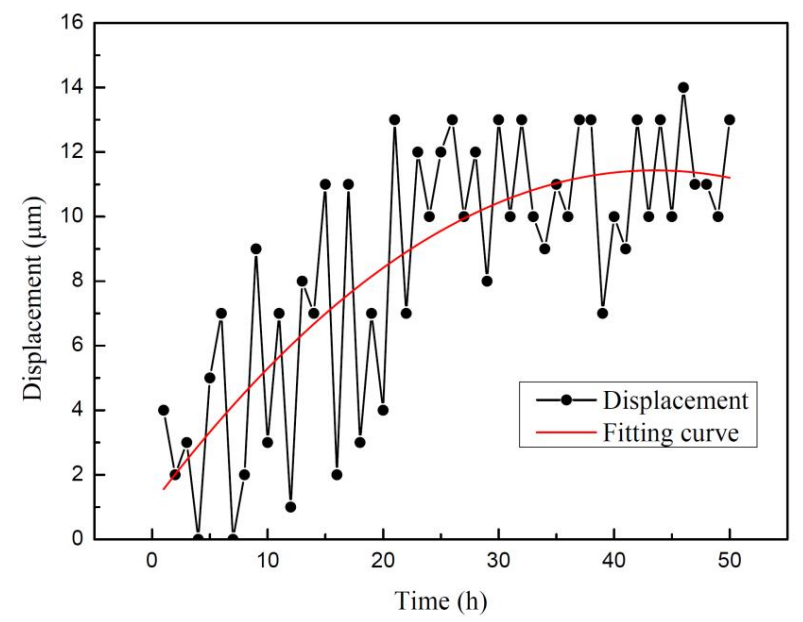

Figure 3. Results of wear displacement

The figure shows the wear displacement value of the friction pairs is also obvious fluctuant. The wear amount of friction pair is increasing over time, but the change tendency gradually become stable. The wear distance of upper specimen is reach to $12 \mu \mathrm{m}$ when the experimental time running to $25 \mathrm{~h}$ and the distance is between $8 \mu \mathrm{m}$ with $13 \mu \mathrm{m}$ for continue to run the rig. Fitting curve in the figure indicates the variation trends of the wear value.
The result of the measurement is different from the equation (4). In that equation, wear depth $\Delta h(\mathrm{t})$ and the time is a function of linear relationship, but the measurement of wear depth is increasing before $25 \mathrm{~h}$ and the tends to be stable for continue to run. The possible reason is that with normal wear before $25 \mathrm{~h}$, the wear formula follows the equation (4); and the debris is produced from the contact surface with running the test, which lubricates the contact pair and reduces the direct contact of the friction pair so as to reduce wear.

\section{Friction and wear coefficient}

The friction coefficient and wear coefficient are calculated by the friction equation and equation (5), and the results are shown in Figure 4. The friction coefficient is shown in left coordinate, the range is 0.2 to 0.8 ; and the wear coefficient in right, the range is $2 \times 10^{-5}$ to $8 \times 10^{-5}$.

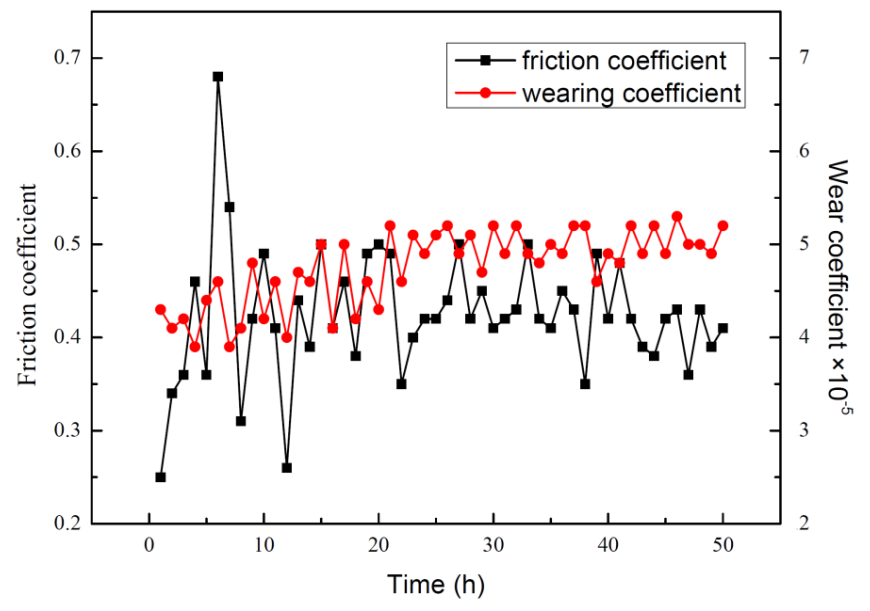

Figure 4. Results of friction and wear coefficient

The change trend of friction coefficient is consistent with the torque measurement, which increases in the initial stage, the maximum value is 0.68 , then gradually tends to be stable, and gradually decreases, with the minimum value of friction coefficient about 0.40 . Wear coefficient also increases with the increase of wear, and gradually tends to be stable, the calculation stability value is about $5 \times 10^{-5}$.

From the results in figure 4, although a large difference in the magnitude of friction and wear coefficient exists, there is still a certain relationship between the friction and wear coefficient. The figure also shown that the time of friction and wear coefficient is basically the same. By using the above mentioned parameters, the equation (6) can be written as:

$$
\frac{K}{\mu}=0.5 \frac{\Delta h(t)}{n T}
$$

Equation (7) shows that the ratio of $K / \mu$ is related with the distance of wear $\Delta h(\mathrm{t})$, the laps of lower Specimen rotation $n$ and the torque $T$. According to the related experiment data and equation (7), the results of friction versus wear coefficient can be obtained in Figure 5. 


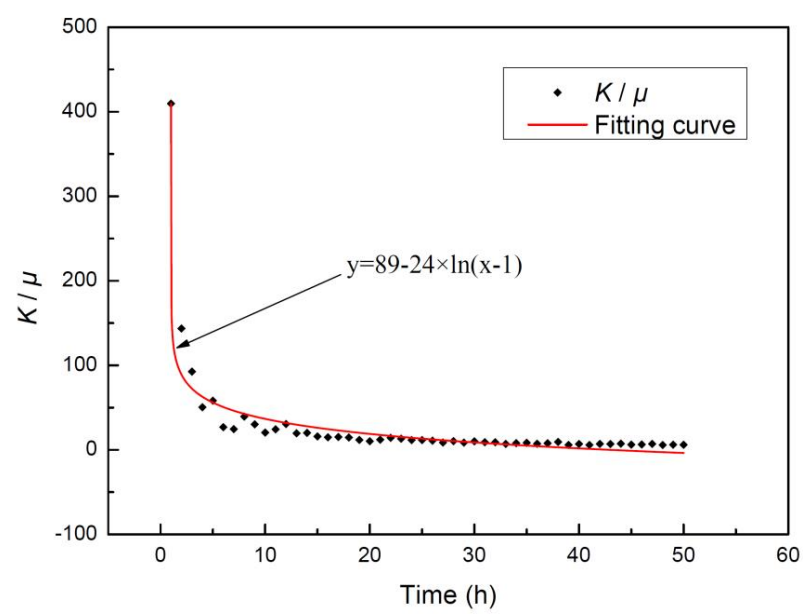

Figure 5. Results of friction versus wear coefficient

In Figure 5, at the startup of test rig, the ratio of $K / \mu$ is large with low value of $n$, but the ratio decreases rapidly and gradually stabilizes with the increasing of $n$. When the motion revolutions accumulated for 21000 laps, the maximum result tends to be 0 . By mathematical fitting, the relationship between the ratio of $K / \mu$ and the time is $y=89-24 \ln (t-1)$, that is, there is a logarithmic relationship between the ratio of $K / \mu$ and the time $t$.

\section{CONCLUSION}

In this paper, the friction performance of the friction pair of aviation door locking device materials 30CrMNSi-LD10CS was investigated by experimental testing and theoretical analysis, and the relationship between friction coefficient and wear coefficient was discussed. The main conclusions in this paper are as follows.

(1) The frictional characteristic of 30CrMNSi-LD10-CS is similar with metallic material. The friction coefficient of $30 \mathrm{CrMNSi}-\mathrm{LD} 10-\mathrm{CS}$ in the measurement of results is not constant, and the maximum value is in the running stage, then the friction coefficient decreases with the time.

(2) The measurement results and theoretical analysis of the wear coefficient are quite different. The experiment has shown that wear coefficient did not follow the linear relationship of time; the wear coefficient increased and tended to be stable with the increase of time.

The results were mainly because after a period of friction, wear debris in the friction had formed a certain layer of lubrication, which reduced wear and the friction coefficient and wear value.
(3) The ratio of friction coefficient and wear coefficient of the designed friction pair has a certain logarithmic relationship with the time.

Subsequent research will discuss the corresponding relationship between the friction coefficients and wear coefficient of the friction pairs with different shapes and different materials, and the theoretical expression of the relationship between the friction and wear of the metal friction pairs can be concluded.

\section{ACKNOWLEDGMENT}

This project is supported by Doctoral Science Foundation of Changsu Institute of Technology (No. KYZ2015054Z).

\section{REFERENCES}

[1] Z. L. Liu, "Principle of Tribology", Higher education Press, Beijing, 2009, pp15-19.

[2] W. X. Liu, "Mechanical reliability design", Tsinghua University Press, Beijing, 2004, pp23-35.

[3] G. T. Rado and H. Suhl, Eds. New York: Academic, 1963, pp. 271-350. T S Eyre, "Wear characteristics of metals", Tribology International, Vol. 9, no. 5, 1976, pp203-212.

[4] P. J. Blau, "Fifty years of research on the wear of metals", Tribology International, Vol. 30, no. 5, 1997, pp321-331.

[5] G. B. V. Kumar, C. S. P. Rao, N. Selvaraj, "Mechanical and Tribological Behavior of Particulate Reinforced Aluminum Metal Matrix Composites - a review", Journal of Minerals \& Materials Characterization \& Engineering, Vol. 10, no. 1, 2011, pp59-91.

[6] Z. Y. Wan, X. Zhao, W. C. Li, "The motion reliability analysis based on the continuous contact "effective length model", and Archard wear", mechanical science and technology, Vol. 5, 2013, pp679-682.

[7] F. P. Bowden and D. Tabor, "The friction and lubrication of solids", Oxford University Press,London, 1950, PP337-354.

[8] J. F. Archard, "Contact and Rubbing of flat surfaces", Journal of Applied Physics, Vol. 24, no. 981, 1953, pp981-984.

[9] J. F. Archard, W. Hirst, "The Wear of Metals Under Unlubricated Conditions", Proceedings of the Royal Society A, Vol 236, no 1206, 1956, pp397-410.

[10] J. F. Archard, "Elastic deformation and the laws of friction", Proceedings of the Royal Society A Mathematical Physical \& Engineering Sciences, Vol. 243, no. 4369, 1957 pp190-205.

[11] R. C. Cozza, D. K. Tanaka, R. M. Souza, "Friction coefficient and abrasive wear modes in ball-cratering tests conducted at constant normal force and constant pressure-Preliminary results", Wear, Vol. 267, no. 1, 2009, pp61-70.

[12] R. C. Cozza, D. K. Tanaka, R. M. Souza, "Friction coefficient and wear mode transition in micro-scale abrasion tests", Tribology International, Vol. 44, no. 12, 2011, pp1878-1889.

[13] R. C. Cozza, "A study on friction coefficient and wear coefficient of coated systems submitted to micro-scale abrasion tests", Surface \& Coatings Technology, Vol. 215, no. 4, 2013, pp224-233. 